\title{
A new modulation scheme for OFDM multitone MFSK over FastTime Varying Channels
}

\author{
Yuelei Xie ${ }^{1, a}$, Yongqiang $\mathrm{Li}^{1, \mathrm{~b}}$, Kewei Han ${ }^{1, \mathrm{c}}$, Shan Ouyang ${ }^{1, \mathrm{~d}}$ \\ ${ }^{1}$ School of information and communication of the Guilin University of Electronic Technology, Guilin, \\ Guangxi, 54100, China \\ aylxie@guet.edu.cn, ${ }^{\text {b}} 624570761 @ q q . c o m,{ }^{d}$ dhmoysh@guet.edu.cn
}

Keywords: OFDM multitone FSK; OFDM-MFSK; spectral efficiency; power efficienc

Abstract. OFDM-MFSK is a robust multicarrier scheme for transmission over fast fading multipath channels. The disadvantage of OFDM-MFSK is a lower spectrum efficiency. In this paper,we investigate the spectrum efficiency of OFDM-MFSK system and proposes a OFDM multitone FSK modulation scheme. The spectrum efficiency can be improved by increasing set of working subcarriers of OFDM in the proposed scheme. Simmulation results show that proposed scheme can achieve a high spectrum efficiency while the power efficiency is still not decreasing.

\section{Introduction}

OFDM has been regarded as the key technology for broadband wireless communication systems. OFDM-MFSK has been proposed by Wetz in [1,2,3] for wireless multi carrier systems over fast fading channel. The OFDM-MFSK is a modulation scheme which combined of m-ary frequency shift keying (MFSK) and OFDM. So it can take advantages of both OFDM and MFSK. And a non-coherent energy detection method can be used at the receiver without channel estimation and equalization. The OFDM-MFSK works very well in combating against frequency and time selective fading while with high power efficiency. However the spectrum efficiency of OFDM-MFSK is so poor that the upper bound of spectrum efficiency is only $0.5 \mathrm{bits} / \mathrm{Hz}$. So it is a major task for OFDM-MFSK to increase the spectrum efficiency.

In $[1,3,4]$, Wetz also proposed a hybrid modulation scheme to increase bandwidth efficiency. The hybrid modulation scheme was named as OFDM-MFSK-DPSK, which jointed DPSK and OFDM-MFSK so as to use the subcarrier phase of OFDM-MFSK symbols to transmit information bits. The bandwidth efficiency can be obviously increased with this hybrid scheme. But the hybrid scheme turns to be very sensitive to frequency selective or fast time variance. In $[5,6]$, a OFDM-N/MFSK scheme was proposed to solve the problem of low spectral efficiency. The scheme employs multiple tone FSK as an alternative to single tone FSK in OFDM-MFSK. So the spectrum efficiency increases significantly on the cost of losing orthogonality among the different N/MFSK vector. However the power efficiency has been decreased greatly. In [7], the performance of coded OFDM-MFSK was improved by using combined alphabets and extended mapping. Compared to un-coded OFDM-MFSK, this method has a higher power efficiency while maintaining the same bandwidth efficiency.

In this paper, we consider the spectrum efficiency as well as the power efficiency for improving OFDM-MFSK system. Then we propose an OFDM multitone FSK scheme, based on [5] and [6], referred to as OFDM-(n+m)/MFSK (in order not to confuse, we put it abbreviated as $(n+m) / M F S K)$. This scheme solves the low spectral efficiency of OFDM-MFSK, and solves the low power efficiency of the traditional OFDM multitone FSK. It also has the certain reference significance in other transmission scheme.

\section{Transmission scheme}

Introduction existing schemes. In order to introduce the principle of OFDM-MFSK, fig.1 gives an example for $M=4$. Only one subcarrier out of 4 is selected (we use solid line indicates it). Note that the 
solid lines represent the carrier that is used for transmission, whereas the dotted lines represent the carriers that are not used for transmission.

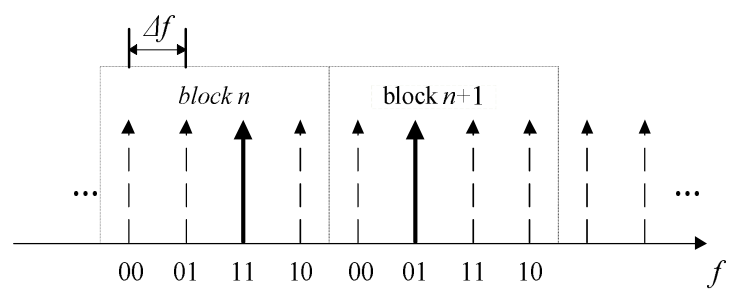

Fig.1 Principle of OFDM-4FSK.

In OFDM-N/MFSK, $\mathrm{N}$ subcarriers out of $\mathrm{M}$ are selected. Because $\mathrm{N}$ have $\left(\begin{array}{c}M \\ N\end{array}\right)$ possibilities, this scheme increases the number of bit per block. At the same time, each block using multiple subcarriers, which can also carry more phase information. The code bits results in the approximated metric $L$ for the $j$ th bit in the $l$ th N/MFSK block can be written as

$$
L_{l, j}=\max _{p \in E_{l, p}^{0}} E_{l, p}-\max _{p \in E_{l, p}^{1}} E_{l, p},
$$

where $E_{l, p}^{0}$ is the set of energies in which bit $j$ is 0 and $E_{l, p}^{1}$ is the set of energies in which bit $j$ is 1 .

As mentioned above, only the amplitude is limited of the selected subcarriers in OFDM-MFSK, and its phase can still be used to transmit information. The phase information are added to form a mixed modulation method of OFDM-N/MFSK. DPSK modulation used on the phase to not affect the non-coherent detection.

Evaluation Criteria of transmission scheme. The spectral efficiency, power efficiency and bit error rate performance are mainly considered to evaluate transmission scheme. Its bit error rate performance can be analyzed spontaneously in the simulation, and spectral efficiency can be shown by the average number of bits per subcarrier, denoted by $\eta_{f}$.

Because the amplitude of selected subcarrier in each grouping is always 1, for ease of illustration and comparison, the energy to transmit one bit data is equal to the average number of subcarriers which it used. It is used as a measure of power efficiency, referred to as $\eta_{p}$ (the smaller the number, the higher the power efficiency).

According to the above definition, the spectral efficiency and power efficiency of OFDM-N / MFSK-DPSK can be expressed as

$$
\begin{gathered}
\eta_{f}=\frac{\left\lfloor\log _{2} C_{M}^{N}\right\rfloor+\alpha N}{M}, \\
\eta_{p}=\frac{N}{\left\lfloor\log _{2} C_{M}^{N}\right\rfloor+\alpha N},
\end{gathered}
$$

where $\alpha=\log _{2} L$, L is phase number of DPSK. OFDM-N/MFSK (without phase information) corresponds to the case of $\mathrm{L}=1$, and OFDM-MFSK corresponds to $\mathrm{N}=1$.

\section{The scheme of OFDM-(n+m)/MFSK}

The basic idea of OFDM-(n+m)/MFSK. The starting point in our OFDM- $(n+m) / M F S K$ scheme is use $\mathrm{M}$ adjacent OFDM subcarriers as a block. And $\mathrm{n}$ or $\mathrm{m}$ subcarriers out of $\mathrm{M}$ are selected. For each subcarrier in the same grouping, the "1" indicates that the subcarrier is actually used in the transmission, and "0" indicates that the subcarrier is not used in transmission. All combinations of 
subcarriers in each group selected are $\sum_{i=0}^{M} C_{M}^{i}=2^{M}$ species. Where $C_{M}^{0}$ indicates that the group is not used and can be assigned to users, thus it can't be used to convey data. The combination of subcarriers can be used are only $2^{M}-1$ kinds of possibilities and can pass (M-1) bit.

The scheme of OFDM- $(\mathrm{n}+\mathrm{m}) / \mathrm{MFSK}$ constitute the mapping table selected $2^{M-1}$ types of frequencies from $2^{M}-1$ types. Its spectral efficiency is $\eta_{f}=\frac{M-1}{M}$. The $2^{M-1}$ kinds of frequency combination can be expressed by Pascal's Triangle (because of $C_{5}^{2}+C_{5}^{3}>2^{5-1}, C_{6}^{2}+C_{6}^{3}>2^{6-1}$, $C_{7}^{3}+C_{7}^{4}>2^{7-1}$ ), see Fig. 2 Pascal's Triangle. But, when $\mathrm{M} \geq 8$, it must use three subcarriers (because of $C_{8}^{3}+C_{8}^{4}<2^{8-1}$, and $\left.C_{8}^{1}+C_{8}^{3}+C_{8}^{4}>2^{8-1}\right)$.

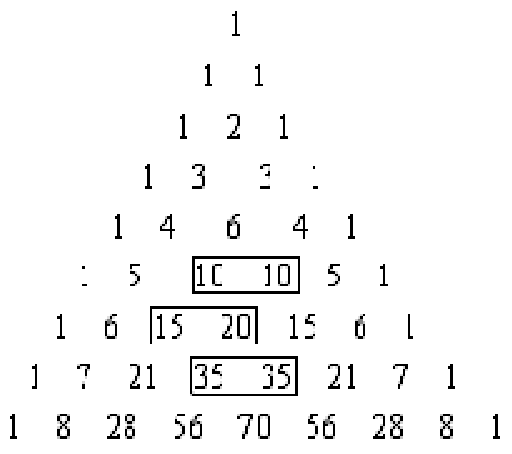

Fig. 2 Pascal's Triangle

The spectral efficiency and power efficiency in different $\mathrm{N}$-nary system combination are shown in Table 1 . Where $\eta_{f} / \eta_{p}$ is the spectral efficiency per power efficiency, the larger the number, the better the system performance. Comprehensive consideration, the case of $M=6$ is the best.

Tab. 1 the performance in different $\mathrm{N}$-nary system combination

\begin{tabular}{|l|l|l|l|l|}
\hline N-nary & best choice & $\eta_{f}$ & $\eta_{p}$ & $\eta_{f} / \eta_{p}$ \\
\hline 2 & 1 & 0.5000 & 1.0000 & 0.5000 \\
\hline 3 & 12 & 0.6667 & 0.6250 & 1.0667 \\
\hline 4 & 12 & 0.7500 & 0.5000 & 1.5000 \\
\hline 5 & 23 & 0.8000 & 0.5938 & 1.3474 \\
\hline 6 & 23 & 0.8333 & 0.5063 & 1.6461 \\
\hline 7 & 34 & 0.8571 & 0.5755 & 1.4894 \\
\hline 8 & 145 & 0.8750 & 0.6005 & 1.4572 \\
\hline 9 & 145 & 0.8889 & 0.6533 & 1.3605 \\
\hline
\end{tabular}

The time-domain expression of mapping symbols in the $i$ th OFDM-(n+m)/MFSK block $s_{i}(t)$ can be written as

$$
s_{i}(t)=\sum_{k \in P_{i}} \exp \left(j \omega_{i, k} t\right) \quad 0 \leq t \leq T_{s},
$$

where $j=\sqrt{-1},\left\{\omega_{i, k}\right\}$ are the angular frequency of non-null subcarriers, and $T_{s}$ is a OFDM symbol period. So, an OFDM symbol can be expressed as 


$$
x(t)=\sum_{i=1}^{N / M} s_{i}(t)=\sum_{i=1}^{N / M} \sum_{k \in P_{i}} \exp \left(j \omega_{i, k} t\right) \quad 0 \leq t \leq T_{s},
$$

where $\mathrm{N}$ is the number of OFDM subcarriers are used, $\mathrm{M}$ is the number of subcarriers per block, $\mathrm{N}$ is an integer multiple of $\mathrm{M}$.

The signal decision criterion. The transmitter or receiver in the high-speed mobile environment, wireless electromagnetic signal have time-varying multipath fading, the typical time-varying multipath fading channel as

$$
h(t, \tau)=\sum_{l=1}^{L} A_{l} \delta\left(\tau-\Delta \tau_{l}\right) \exp \left(j \omega_{l} t\right),
$$

where $\mathrm{L}$ is the number of multipath, $A_{l}$ is the time varying complex fading coefficient in the first path, $\omega_{l}$ is Doppler frequency offset in the first path, and $\Delta \tau_{l}$ is the path delay in the first path respect to the reference path. So, the signal in receiver $r(t)$ can be expressed as

$$
\begin{aligned}
& r(t)=\sum_{l=1}^{L} A_{l} x\left(t-\Delta \tau_{l}\right) \exp \left(j \omega_{l} t\right)+w(t)=\sum_{l=1}^{L} \sum_{i=1}^{N / M} \sum_{k \in P_{i}} A_{l} \exp \left\{j \omega_{i, k}\left(t-\Delta \tau_{l}\right)\right\} \exp \left(j \omega_{l} t\right)+w(t) \\
& =\sum_{i=1}^{N / M} \sum_{k \in P_{i}} \sum_{l=1}^{L} A_{l} \exp \left(j \omega_{l} t\right) \exp \left\{j \omega_{i, k}\left(t-\Delta \tau_{l}\right)\right\}+w(t) \quad 0 \leq t \leq T_{s},
\end{aligned}
$$

where $w(t)$ is white Gaussian noise. Corresponding to the $i$ th block in receiver, the signal $y_{i}(t)$ can be written as

$$
\begin{aligned}
y_{i}(t) & =\sum_{k \in P_{i}} \sum_{l=1}^{L} A_{l} \exp \left(j \omega_{l} t\right) \exp \left\{j \omega_{i, k}\left(t-\Delta \tau_{l}\right)\right\}+w_{i}(t) \\
& =\sum_{k \in P_{i}} \sum_{l=1}^{L} A_{l} \exp \left(j \omega_{l} t\right) \exp \left(j \omega_{i, k} t\right) \exp \left(-j \omega_{i, k} \Delta \tau_{l}\right)+w_{i}(t) \\
& =\sum_{k \in P_{i}} \sum_{l=1}^{L} A_{l} \exp \left\{j\left(\omega_{l}+\omega_{i, k}\right) t\right\} \exp \left(-j \omega_{i, k} \Delta \tau_{l}\right)+w_{i}(t) \quad 0 \leq t \leq T_{s},
\end{aligned}
$$

where $w_{i}(t)$ is band-limited white Gaussian noise. After FFT, the formula can be written as

$$
Y_{i}(\omega)=2 \pi \sum_{k \in P_{i}} \sum_{l=1}^{L} A_{l} \delta\left(\omega-\omega_{i, k}-\omega_{l}\right) \exp \left(-j \omega_{i, k} \Delta \tau_{l}\right)+W_{i}(\omega)
$$

In the $i$ th OFDM-OFDM block, each subcarrier energy $E_{i, k}$ can be expressed as

$$
E_{i, k}=\frac{1}{2 \pi} \int_{\omega_{i, k}-\Delta \omega / 2}^{\omega_{i, k}+\Delta \omega / 2}\left|Y_{i}(\omega)\right|^{2} d \omega
$$

where $E_{i, k}$ is the $k$ th subcarrier energy in $i$ th block, $\Delta \omega$ is the subcarrier spacing, assuming $\left|\omega_{l}\right|<\Delta \omega / 2$, then 


$$
E_{i, k}=\left\{\begin{array}{ll}
\left|\sum_{l=1}^{L} A_{l} \exp \left(-j \omega_{i, k} \Delta \tau_{l}\right)\right|^{2}+E_{W\left(\omega_{i, k}\right)} & k \in P_{i} . \\
E_{W\left(\omega_{i, k}\right)} & k \notin P_{i}
\end{array} .\right.
$$

The process of signal decision including the following:

a) $E_{i, k}, k=1,2,3, \mathrm{~L} 6$ is arranged in descending order to obtain $E_{i, k_{j}}, j=1,2,3, \mathrm{~L} 6$.

b) We let $\hat{s}_{i, k_{j}}=\left\{\begin{array}{ll}1 & j=1,2,3 \\ 0 & j=4,5,6\end{array}\right.$, where $\hat{s}_{i, k}$ is the signal decision result of the $k$ th subcarrier in $i$ th group.

c) If $\left\{\hat{s}_{i, k}: k=1,2,3, \mathrm{~L} 6\right\}$ equal to $100110 、 110100$ and 111000 (Tab.2 does not appear) or $E_{i, k_{3}}<K \cdot E_{i, k_{2}}$, then $\hat{s}_{i, k_{3}}=0$, where $\mathrm{K}$ is a positive number less than 1 . The value of $\mathrm{K}$ has a certain effect on the bit error rate performance, and the appropriate value of $\mathrm{K}$ will be analyzed in Figure 8 and 9.

\section{Simulation Results}

We make the carrier frequency as $\mathrm{fc}=5.8 \mathrm{GHz}$, the subcarrier number as $\mathrm{Nf}=256$, the useful subcarrier as Nfused $=162$, the spacing of subcarrier as $\Delta \mathrm{f}=312.5 \mathrm{kHz}$, the cyclic prefix as $T_{g}=$ $0.8 \mu \mathrm{s}$, the cycle of the whole OFDM symbol Ts $=4 \mu \mathrm{s}$. In our simulation, we use the convolutional code which symbol rate is $1 / 2$, and its generating polynomial is [133,171]

Figure 4 shows the overall bit error rate performance of the mix modulation is lower than the single modulation. where 4F, 3/4F, (2+3)/6F and 2D were shorthand of 4FSK, 3/4FSK, (2+3)/6FSK and 2DPSK. The (2+3)/6FSK-2DPSK bit error rate performance is similar to the 3/4FSK-2DPSK, and the $(2+3) / 6$ FSK bit error rate performance is similar to 3/4FSK. They, said all above, are lower than 4FSK and 4FSK-2DPSK. Because the DPSK modulation is loaded on the single modulation (FSK), and it correct demodulation depends on the FSK correct demodulation. The $(2+3) / 6$ FSK and $(2+3) / 6$ FSK-2PSK advantage of spectral efficiency and power efficiency, can be found in Tab. 3 .

Tab. 2 The spectral efficiency and power efficiency of each scheme

\begin{tabular}{ccc}
\hline modulation scheme & $\eta_{f}$ & $\eta_{p}$ \\
\hline 4FSK & 0.5 & 0.5 \\
3/4FSK & 0.5 & 1.5 \\
(2+3)/6FSK & 0.8333 & 0.5062 \\
4FSK-2DPSK & 0.75 & 0.333 \\
3/4FSK-2DPSK & 1.25 & 0.6 \\
(2+3)/6FSK-2DPSK & 1.2552 & 0.3361 \\
\hline
\end{tabular}




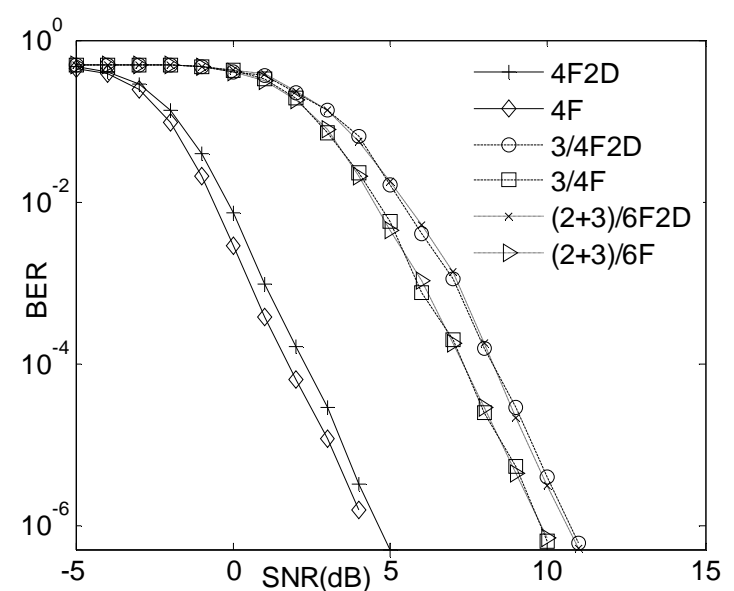

Fig.3 Bit error curve of each scheme in Gauss white noise channel

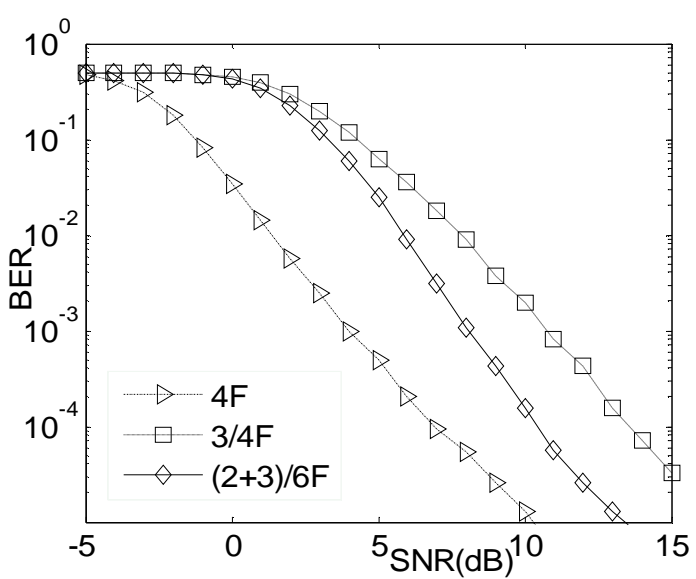

Fig.5 Bit error rate performance of FSK part

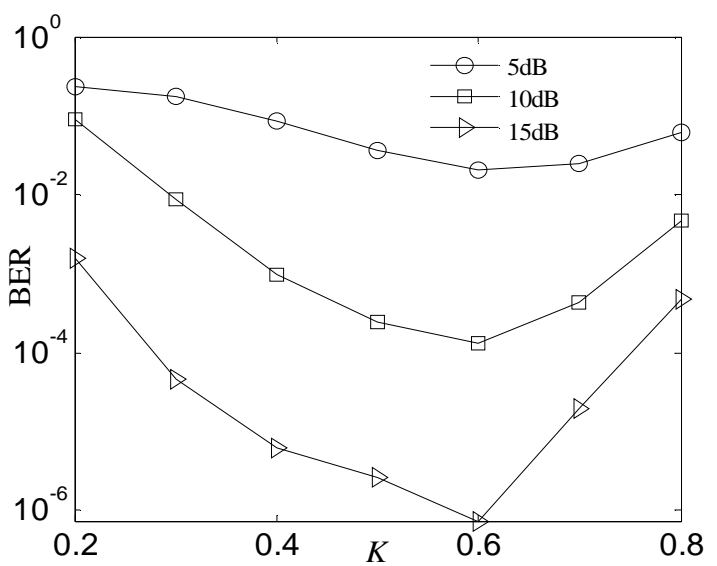

Fig.7 K with doubly selected channel

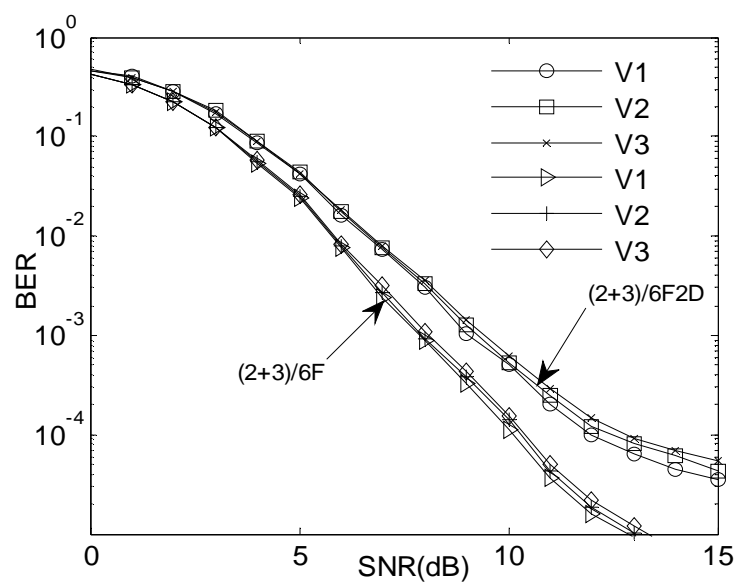

Fig.4 Bit error rate performance at different speeds

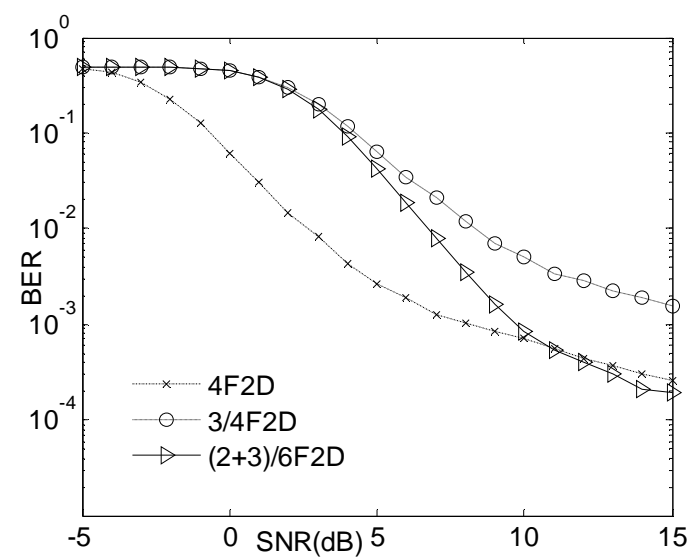

Fig.6 Bit error rate performance of the mix modulation.

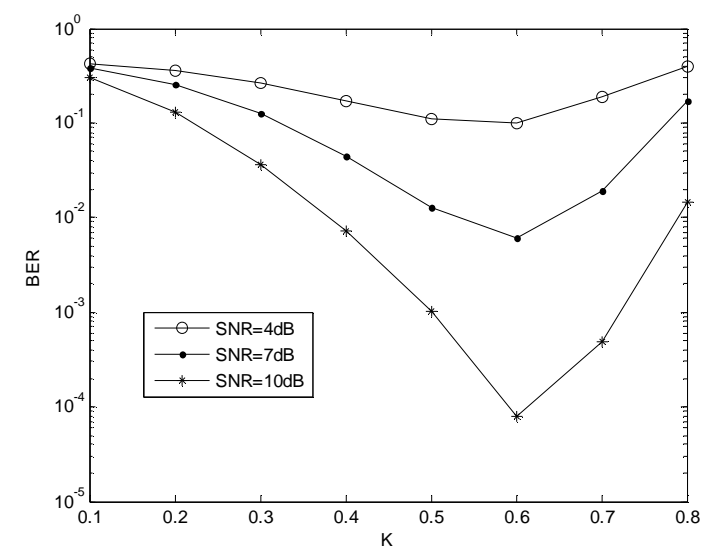

Fig.8 K with white Gaussian noise channel

Fig. 5 shows the bit error rate performance of $(2+3) / 6 \mathrm{FSK}$ is insensitive to the speed of the communication terminal removing, where $V 1, \mathrm{~V} 2$ and $\mathrm{V} 3$ respectively represent $0 \mathrm{~km} / \mathrm{h}, 300 \mathrm{~km} / \mathrm{h}$ and $600 \mathrm{~km} / \mathrm{h}$. It indicates that it is robust to the channel of the fast time-varying, and is suitable for wireless communication in high speed mobile environment. Only $600 \mathrm{~km} / \mathrm{h}$ is simulated in the following simulation.

Fig. 6 shows the bit error rate performance of the (2+3)/6FSK is better than the 3/4FSK, and the 4FSK is better than the (2+3)/6FSK. It is less affected by the fading. Because the former in each group have more subcarriers are zero. 
Fig. 7 shows the bit error rate performance of the $(2+3) / 6$ FSK-2DPSK is better than the 3/4FSK-2DPSK. This is because the average frequency interval of the subcarriers used in the latter is larger, and its DPSK part will have a severely impact by the frequency selective fading.

The best value of $\mathrm{K}$ can find through simulation. The simulation results in the white Gaussian noise channel and doubly selected channel are shown in Fig. 8, 9. It can be seen that $\mathrm{K}=0.6$ is the best choice (in $(1+2 / 4 \mathrm{FSK}), \mathrm{K}=0.2$ is the best choice). The optimal value of $\mathrm{K}$ in the OFDM- $(n+m) / M F S K$ system can be obtained, fixing three parameters of $n, m, M$.

\section{Conclusions}

This paper presents a new OFDM multitone MFSK modulation scheme, which uses non-coherent detection without channel estimation. Compared with the previous OFDM multitone FSK scheme, its performance of spectrum efficiency, power efficiency and bit error rate are improved in different degree. Its comprehensive performance is better than that of the existing schemes. Simmulation results show that proposed scheme can achieve a high spectrum efficiency while the power efficiency is still not decreasing. It is suitable for fast fading frequency selective channel, and can realize the wireless communication in high speed mobile environment.

\section{Acknowledgements}

This work was financially supported by the National Natural Science Foundation of China(61461015), Guangxi Natural Science Foundation（2015GXNSFAA139302）.

\section{References}

[1] Wetz M, Perisa I, Teich W G, et al: Wireless Personal Communications. 2008, 47(1):113-123.

[2] M.Wetz: Transmission Methods for Wireless Multi Carrier Systems in Time-Varying Environments, PhD thesis, University of Ulm, Germany, October 2011,DerandereVerlag.

[3] Peiker-Feil, Eva, et al: "OFDM-MFSK as a Special Case of Noncoherent Communication Based on Subspaces." Ofdm 2012, International Ofdm Workshop 2012:1-5.

[4] Wetz M, Periša I, Teich W G, et al: OFDM-MFSK with differentially encoded phases for robust transmission over fast fading channels[C]// in Proc. $11^{\text {th }}$ International OFDM-Workshop, Hamburg, Germany, August 2006.

[5] Linduska A: Analysis of OFDM Multitone FSK Schemes in Frequency Selective Fast Fading Channels $[\mathrm{C}] / / 9^{\text {th }}$ International Symposium on Communication Theory and Applications (ISCTA), July 2007, Ambleside, England.

[6] Peiker E, Yammine G, Teich W G, et al: Increasing the Bandwidth Efficiency of OFDM-N/MFSK[C]// Ofdm 2014; International Ofdm Workshop. VDE, 2014.

[7] Yammine G, Peiker E, Teich W G, et al: Improved performance of coded OFDM-MFSK using combined alphabets and extended mapping[C]// International Symposium on Turbo Codes and Iterative Information Processing. IEEE, 2014:17-21. 